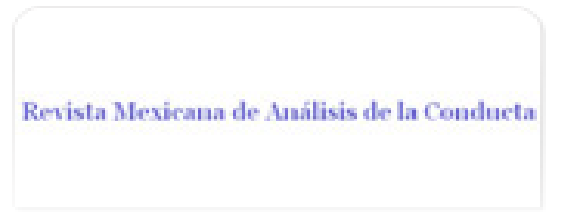

Revista Mexicana de Análisis de la Conducta ISSN: 0185-4534

editora@rmac-mx.org

Sociedad Mexicana de Análisis de la Conducta México

ROCA, ALICIA; BRUNER, CARLOS A.

Un análisis del origen del consumo excesivo de agua del beber inducido por el programa Revista Mexicana de Análisis de la Conducta, vol. 37, núm. 2, 2011, pp. 177-204

Sociedad Mexicana de Análisis de la Conducta Guadalajara, México

Disponible en: http://www.redalyc.org/articulo.oa?id=59319255011

- Cómo citar el artículo

- Número completo

- Más información del artículo

- Página de la revista en redalyc.org

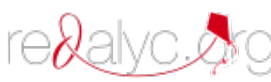

Sistema de Información Científica Red de Revistas Científicas de América Latina, el Caribe, España y Portugal Proyecto académico sin fines de lucro, desarrollado bajo la iniciativa de acceso abierto 


\title{
UN ANÁLISIS DEL ORIGEN DEL CONSUMO EXCESIVO DE AGUA DEL BEBER INDUCIDO POR EL PROGRAMA
}

\author{
AN ANALYSIS OF THE ORIGIN OF EXCESSIVE WATER INTAKE \\ OF SCHEDULE-INDUCED DRINKING
}

ALICIA ROCA Y CARLOS A. BRUNER

Universidad Nacional Autónoma de México, Facultad de Psicología, Laboratorio de Condicionamiento Operante, México, D.F., México

\begin{abstract}
Resumen
El propósito del estudio fue analizar el origen del consumo excesivo de agua durante las sesiones de beber inducido por el programa (BIP). Tres ratas vivieron dentro de las cámaras experimentales y se registró el consumo de agua continuamente. En el Experimento 1 se distribuyeron tres sesiones de BIP en cada período de 24 horas, en las que se entregaron uno, tres u ocho gramos de comida conforme a un programa de tiempo fijo $180 \mathrm{~s}$. El consumo diario de agua se concentró dentro de las sesiones y el volumen de agua consumida fue proporcional a la cantidad de comida entregada. En el Experimento 2 se presentaron las bolas de comida juntas al inicio de cada sesión de BIP y en una segunda condición se entregaron las bolas de comida juntas al inicio de cada día. El volumen de agua consumida permaneció constante durante cada período de 24 horas a través de los dos experimentos del estudio, independientemente de la distribución temporal de la comida. Se concluyó que el consumo de agua durante las sesiones de BIP no es excesivo. La privación de comida y la entrega de comida a intervalos son conducentes a confinar el beber durante las sesiones.
\end{abstract}

Los experimentos del artículo forman parte de la tesis doctoral del primer autor, la cual fue merecedora a la Medalla Alfonso Caso de la UNAM. El segundo autor fue el director de la tesis. Dirigir comentarios al primer autor. Laboratorio de Condicionamiento Operante de la Facultad de Psicología, Universidad Nacional Autónoma de México, Av. Universidad 3004, Col. Copilco - Universidad. C.P. 04510, Del. Coyoacán, México, D.F. Correo electrónico: alicia.roca@live.com 
Palabras clave: Beber inducido por el programa, períodos de observación de 24 horas, interacción entre privaciones de comida y agua, distribución temporal del consumo de agua, ratas.

\begin{abstract}
)
The purpose of the study was to analyze the origin of excessive water intake during schedule-induced drinking (SID) sessions. Three rats lived within the experimental chambers and water intake was continuously recorded. In Experiment 1, three onehour SID sessions were intruded within each 24-hour period, in which one, three, or eight grams of food were delivered on a 180-s fixed time schedule. Daily water intake remained confined within the sessions, and the volume of water that each rat drank was proportional to meal size. Holding constant the meal size, in Experiment 2 the food pellets were delivered at the beginning of each SID session and in a second condition, at the beginning of each 24-hour period. Volume intake remained constant throughout the two experiments, regardless of food distribution. Results suggest that the SID procedure does not generate excessive water intake. Food deprivation and spaced-food delivery enhance the concentration of the rats' daily water intake within the sessions.
\end{abstract}

Key words: Schedule-induced drinking, 24-hour observation periods, food and water deprivation interaction, temporal distribution of water intake, rats.

Existe un procedimiento conducente a hacer que las ratas privadas únicamente de comida consuman cantidades sustanciales de agua. Este procedimiento consiste en exponer a las ratas privadas de alimento a un programa intermitente de comida y permitir el libre acceso a una fuente de agua. Por ejemplo, Falk (1961) restringió el alimento a las ratas dentro de sus cajas habitación hasta que alcanzaron el $80 \%$ de su peso ad libitum pero les permitió el acceso libre al agua. Posteriormente, las colocó en una cámara experimental en la cual había una palanca y un bebedero con agua. Las ratas presionaban la palanca para obtener bolas de alimento conforme a un programa de intervalo variable (IV) $60 \mathrm{~s}$. Las ratas bebían agua después de obtener cada bola de comida y la cantidad de agua que bebieron fue aproximadamente 29 $\mathrm{ml}$ de agua por hora durante cada una de las sesiones experimentales, las cuales tenían una duración de 3.17 horas. Debido a que los sujetos únicamente bebían aproximadamente $1 \mathrm{ml}$ de agua por hora en sus cajas habitación en las que tenían acceso libre a la comida y al agua antes de exponerlas al experimento, el consumo global de agua durante las sesiones era excesivo. Falk le llamó a éste fenómeno polidipsia inducida por el programa.

Después del estudio de Falk (1961) surgieron varios estudios en los que se determinaron las condiciones bajo las cuales ocurría la polidipsia. Se encontró que el consumo excesivo de agua no sólo ocurría cuando las ratas tenían que emitir 
una respuesta, como presionar una palanca, para obtener la comida sino también cuando las bolas de alimento se entregaban a intervalos de tiempo independientemente de la conducta de los sujetos (ver Christian, Schaeffer \& King, 1977 para una revisión de los programas de entrega de comida empleados). Los experimentos en los cuales se mostró que el consumo de agua disminuía considerablemente cuando se exponía a los sujetos a un programa de comida de reforzamiento continuo (CRF) o bien cuando la comida estaba disponible continuamente en un recipiente dentro de la cámara experimental desde el inicio de la sesión llevó a la conclusión de que la intermitencia de la entrega de comida era una condición necesaria para producir polidipsia (e.g., Falk, 1961; King, Mc Gill, Pierson, \& Schaeffer, 1972).

Además del espaciamiento temporal de la comida, se consideró que la privación de comida era una segunda condición necesaria para que ocurriera la polidipsia (Falk, 1969; 1971). Esta conclusión está basada en el hallazgo de que aumentar el peso de las ratas entregándoles progresivamente una mayor cantidad de alimento en sus cajas habitación resulta en disminuciones progresivas en el volumen de agua consumida durante las sesiones experimentales, hasta el punto en el que el consumo de agua es muy bajo cuando las ratas alcanzan el $100 \%$ de su peso en alimentación libre o bien cuando la comida está disponible continuamente dentro de las cajas habitación de los sujetos (e.g., Freed \& Hymowitz, 1972; Roper \& Nieto, 1979). En la mayoría de los estudios las ratas se han mantenido al $80 \%$ de su peso en alimentación libre restringiendo la cantidad de comida entregada en las cajas habitación.

Existen variaciones en la cantidad de agua que las ratas beben a través de los diferentes experimentos que están reportados en la literatura. Estas variaciones en el consumo de agua están determinadas por diferentes parámetros, que incluyen la cantidad de comida entregada por ocasión durante las sesiones experimentales, el nivel de privación de comida y el intervalo entre entregas de comida sucesivas. En reconocimiento a que el volumen de agua consumida puede variar dependiendo de éstos parámetros y a que el consumo de agua se debe al espaciamiento temporal de la comida, el término de polidipsia se sustituyó por beber inducido por el programa (Staddon, 1977). A pesar de que el consumo de agua puede variar conforme a los parámetros de los experimentos, los estudios publicados sobre beber inducido por el programa (en adelante, BIP) muestran que la presentación de comida a intervalos de tiempo a ratas privadas de alimento resulta en un aumento considerable en el consumo de agua relativo a la cantidad de agua que las ratas beben normalmente. En una revisión de los experimentos publicados sobre BIP, la mayoría de las sesiones experimentales tienen una duración de entre una y tres horas, y se ha reportado que la cantidad de agua que las ratas beben durante estas sesiones relativamente cortas iguala o excede el consumo de agua que ocurre durante un período de 24 horas (e. g., Falk, 1961).

Existen varias teorías conforme a las cuales se intentó explicar el consumo excesivo de agua durante las sesiones de BIP. Muchas de estas teorías están basadas en la típica distribución temporal de los episodios de beber dentro del intervalo entre 
entregas de comida durante las sesiones. Por ejemplo, Falk (1966a; 1966b), Lotter, Woods y Vaselli (1973) y Stein (1964) encontraron que la mayor parte del consumo de agua ocurría inmediatamente después de cada entrega de comida, por lo que algunos autores sugirieron que el BIP se debía simplemente a que las bolas de alimento que se usaban en las sesiones resecaban la boca de las ratas y por lo tanto les producían sed (e.g., Stein, 1964). Esta explicación se descartó debido a que las ratas seguían consumiendo el agua a pesar de que durante las sesiones se les entregaba alimento húmedo (e. g., Falk, 1971). Una segunda explicación que se ofreció sobre la ocurrencia del BIP era que cada entrega de comida señalaba un período de baja probabilidad de recibir más comida, por lo que las ratas bebían agua por la limitación de alternativas conductuales dentro de la cámara experimental. Para probar la pertinencia de esta explicación, en algunos estudios se emplearon programas de segundo orden en los cuales se presentaron señales en sustitución de algunas bolas de comida (e.g., Allen, Porter, \& Arazie, 1975; Rosenblith, 1970). Por lo tanto, si efectivamente las ratas bebían agua para "llenar el tiempo" entre reforzadores de comida sucesivos, entonces las señales producirían beber, dado que señalaban una baja probabilidad de recibir más bolas de alimento. No obstante, los resultados de los experimentos en los que se emplearon programas de reforzamiento de segundo orden fueron contradictorios entre los diferentes estudios.

Otra explicación sobre la ocurrencia del BIP, fue que el consumo de agua era reforzado accidentalmente por la entrega de la comida. Por ejemplo, Clark (1962) sugirió que si bien los animales beben agua después de la entrega de la comida, los períodos de beber podían extenderse hasta ocurrir en contigüidad con la comida subsecuente y por lo tanto, la conducta de beber agua era reforzada accidentalmente. Algunos autores impusieron demoras entre los lengüetazos para obtener agua y la entrega de la comida con el propósito de determinar si el consumo de agua disminuiría cuando la entrega de la comida no ocurría en contigüidad temporal con el consumo de agua (e. g., Falk, 1964; Pellón \& Blackman, 1987). A pesar de que añadir la demora entre el consumo de agua y la entrega de la comida resulta en disminuciones en el consumo de agua, el BIP no pudo explicarse en términos de reforzamiento supersticioso, dado que las ratas siguen consumiendo el agua sustancialmente aún bajo estas condiciones.

La falta de explicaciones sobre el origen del consumo sustancial de agua durante las sesiones de BIP basadas en pruebas empíricas resultó en la elaboración de constructos teóricos para explicar el fenómeno. Por ejemplo, Falk (1977) comparó al BIP con las conductas de desplazamiento debido a que el consumo de agua ocurría consistentemente cuando se impedía que el animal tuviera acceso a la comida durante un período de tiempo. Timberlake $(1997,2000)$ afirmó que la presentación de comida a intervalos controlaba la activación de un mecanismo regulatorio específico de las ratas que resultaba en el consumo de agua.

Las diferentes explicaciones propuestas para explicar el BIP tienen la similitud de que enfatizan en la comida como el reforzador presente durante las sesiones 
experimentales, debido a que las ratas están privadas de comida y tienen acceso al agua continuamente, tanto en sus cajas habitación como en las sesiones experimentales. Por lo tanto, se consideró al conusmo de agua como un efecto anómalo que resultaba de espaciar la comida temporalmente. No obstante, existe evidencia de que la privación de comida resulta en disminuciones importantes en el consumo de agua, en otras palabras, la privación de comida resulta en una privación indirecta de agua (Bolles, 1961; López-Espinoza \& Martínez, 2005; Verplanck \& Hayes, 1953). Por ejemplo, Bolles reportó que después de privar a ratas de comida durante $23 \mathrm{hr}$, las ratas disminuyeron su consumo de agua en un $60 \%$ respecto a una condición en la que tenían agua y comida disponibles continuamente. El hecho de que la privación de comida controle una disminución considerable en el consumo de agua respecto a una condición de alimentación libre, implica que en el procedimiento de BIP al restringir el acceso a la comida en las cajas habitación de las ratas, también se está produciendo indirectamente una privación de agua.

Si bien bajo el procedimiento de BIP la privación de comida resulta en una privación indirecta de agua en las cajas habitación de las ratas, la siguiente pregunta que surge es por qué la entrega de comida a intervalos durante las sesiones controla que las ratas beban agua excesivamente durante las sesiones. Un segundo hallazgo en la literatura sobre el consumo de comida y agua en ratas que es pertinente para la contestación a esta pregunta es que si bien la privación de comida resulta en una disminución considerable en el consumo de agua, una vez que se entrega comida a las ratas que habían estado privadas de comida, se restablece el consumo de agua (Fitzsimons \& Le Magnen, 1969; Verplanck \& Hayes, 1953). Por ejemplo, Fitzsimons y Le Magnen encontraron que a pesar de que las ratas tenían acceso al agua continuamente durante períodos de 24 horas, las ratas bebían el 70\% de su ración diaria de agua durante las únicas dos horas del día en las que los autores permitían a los sujetos el acceso a la comida.

Conforme a los resultados de los estudios sobre comer y beber en ratas relativos a la privación indirecta de agua que resulta de la privación de comida y el posterior restablecimiento del consumo de agua, es factible que el procedimiento tradicional de BIP, que involucra restringir la comida a las ratas en sus cajas habitación y posteriormente entregarles comida a intervalos de tiempo en las cámaras experimentales sea una forma de garantizar que los sujetos beban la mayor parte de su ración diaria de agua durante las sesiones experimentales, lo que explicaría el consumo aparentemente "excesivo" durante las sesiones. En la mayoría de los estudios de BIP reportados en la literatura, se registró el consumo de agua exclusivamente durante las sesiones experimentales de una duración relativamente corta (de una a tres horas) y posteriormente se colocaba a los sujetos en las cajas habitación, en las cuales se les entregaba a las ratas la cantidad complementaria de comida para mantenerlas en el nivel de privación especificado.

Para probar la hipótesis de que el beber aparentemente excesivo se debía a una redistribución del consumo diario de agua al momento en el que está vigente 
la sesión de BIP, Roca y Bruner (2007) colocaron a ratas privadas de comida en cámaras experimentales individuales durante períodos de 24 horas. Las cámaras experimentales contenían un bebedero con agua y se registraron los lengüetazos al tubo del bebedero durante todo el período de 24 horas. Roca y Bruner programaron una sesión de BIP de una hora de duración dentro de un período de observación del consumo de agua de 24 horas, la cual consistía en entregar bolas de alimento conforme a un programa de TF $180 \mathrm{~s}$. En el resto del período de 24 horas no se entregó comida. Cada período de observación de 24 hr dentro de la cámara experimental se intercaló con un período de 24 horas en el que las ratas permanecieron en su caja habitación y sólo se les entregaba la cantidad necesaria de comida para mantenerlas al $80 \%$ de su peso ad libitum. Roca y Bruner encontraron que las ratas consumieron alrededor del $30 \%$ de su consumo diario de agua durante la hora en la que estuvo vigente la sesión de BIP en los días en los que las ratas permanecían en las cámaras experimentales. El máximo número de lengüetazos al tubo se observó durante los subintervalos del período de 24 horas en los que estuvo vigente la sesión de BIP. Para mantener a las ratas en el peso especificado era necesario entregar una cantidad considerablemente mayor durante los días en los que las ratas permanecían en sus cajas habitación que durante los días en los que permanecían en las cámaras experimentales. Se observó que el volumen de agua consumida dentro de las cajas habitación fue mayor que durante los días en los que permanecieron en las cámaras experimentales. Los resultados de Roca y Bruner sugieren que el aspecto del procedimiento de intercalar días en las cámaras experimentales con días en las cajas habitación, lo cual involucró entregar cantidades diferentes de comida, determinó una aparente redistribución del consumo de agua durante cada período de $48 \mathrm{hr}$, de tal forma que las ratas consumieron más agua en donde recibían la mayor parte de su alimento (i. e., la caja habitación).

En los dos experimentos que conforman el presente estudio tres ratas privadas de comida vivieron dentro de las cámaras experimentales por un total de 90 días y se registraron los lengüetazos a un bebedero con agua continuamente. En ambos experimentos se distribuyeron cuatro sesiones de BIP de una hora durante cada período de observación de 24 horas. En el Experimento 1 del presente estudio, se determinaron los efectos de entregar diferentes cantidades de comida sobre el volumen de agua consumida y sobre la distribución temporal del consumo de agua durante cada período de 24 horas (antes, durante y después de las sesiones de BIP). En el Experimento 2 del presente estudio se intentó esclarecer el papel del espaciamiento temporal de la comida durante las sesiones de BIP en el consumo excesivo de agua. Si la entrega de comida durante las sesiones restablece el consumo de agua, es incierto por qué en los estudios de BIP se ha reportado que el consumo excesivo de agua no ocurre cuando se entrega la comida toda junta al inicio de la sesión experimental o bien cuando el intervalo entre entregas de alimento es menor a 30 s (e. g., Falk, 1969; King et al. 1972). Por lo tanto, en el Experimento 2 del presente estudio se compararon los efectos de la entrega simultánea de comida y de la entrega 
de comida a intervalos de tiempo sobre el volumen de agua consumida y sobre la distribución temporal del consumo de agua antes, durante y después de las sesiones de BIP. El propósito general del presente trabajo fue analizar el origen del consumo excesivo de agua de las ratas durante las sesiones de BIP.

\section{Experimento 1}

En los estudios sobre consumo de comida y de agua en ratas se mostró que privar a las ratas de comida resulta en una disminución en el volumen de agua consumida. Complementariamente, al restablecer la comida se restablece el consumo de agua (e. g., Bolles, 1961; Verplanck \& Hayes, 1953). Conforme a estos dos fenómenos se hipotétizó que la función de la comida a intervalos bajo el procedimiento de BIP era la de restablecer el consumo de agua, lo cual explicaría su aparente carácter sustancial. Si la función de la comida a intervalos durante las sesiones de BIP es la de restablecer el consumo de agua, se esperaría que a mayor cantidad de comida entregada, mayor sería el aumento en el volumen de agua consumida durante las sesiones. El propósito del Experimento 1 fue determinar los efectos de variar la cantidad de comida durante las sesiones de BIP sobre la distribución temporal y sobre el volumen de agua que consumen las ratas durante cada período de 24 horas. Para cumplir con el propósito del experimento, la estrategia que se empleó fue exponer a los sujetos a cuatro sesiones de BIP de una hora cada una en las que se entregaron diferentes cantidades de comida conforme a un programa de TF $180 \mathrm{~s}$.

Método

\section{Sujetos}

Se utilizaron tres ratas macho Wistar de tres meses de edad al inicio de la investigación y experimentalmente ingenuas. Se privó a las ratas al $80 \%$ de su peso ad libitum pero tuvieron acceso libre al agua durante todo el experimento.

\section{Aparatos}

Se emplearon tres cámaras experimentales de $23 \mathrm{~cm}$ de alto por $24 \mathrm{~cm}$ de largo y 26 $\mathrm{cm}$ de fondo. Las paredes y el techo de las cámaras se construyeron con Plexiglas $₫$ y el panel frontal era de aluminio. Cada cámara experimental se colocó dentro de un cubículo sonoamortiguado de madera equipado con un ventilador. En el panel frontal de cada cámara, a $8.5 \mathrm{~cm}$ del piso y a $5 \mathrm{~cm}$ de la pared izquierda se colocó un tubo de metal con una apertura de $0.7 \mathrm{~cm}$ de diámetro. El tubo estaba conectado a una botella con agua que se encontraba en la parte posterior del panel. Se usó un contador de lengüetazos (Med Assoc @ ${ }^{\circledR}$., Modelo ENV-250A) conectado a la parte posterior del tubo. A $14 \mathrm{~cm}$ a la derecha del tubo de metal, se colocó un recipiente para bolas de comida que medía $5 \mathrm{~cm}$ de ancho, $5 \mathrm{~cm}$ de largo y sobresalía $6 \mathrm{~cm}$ dentro de la cámara. La parte posterior del recipiente para la comida se conectó 
mediante una manguera a un dispensador para bolas de comida (Med Assoc $\mathbb{R}$., Modelo ENV-203). Las bolas de comida pesaban $25 \mathrm{mg}$ y se fabricaron remoldeando polvo de comida para ratas marca Harlan-Teklad(®. Al centro de la parte superior de la pared frontal, a $18.5 \mathrm{~cm}$ del piso de cada cámara, se colocó un foco de $28 \mathrm{v}$ que proporcionó iluminación general. Los eventos experimentales se controlaron y registraron con una computadora con software Med-Pc ${ }^{\circledR}$ conectada a las cajas experimentales por medio de una interfaz Med Associates ${ }^{\circledR}$

\section{Procedimiento}

Se registró el peso de las tres ratas en alimentación libre durante 10 días y posteriormente se les limitó la cantidad de comida dentro de las cajas habitación hasta que alcanzaron el $80 \%$ de su peso ad libitum. Una vez que las ratas alcanzaron el $80 \%$ de su peso ad libitum inició el experimento, en el cual las ratas permanecieron diariamente en las cámaras experimentales. El tubo del bebedero con agua estuvo disponible continuamente dentro de las cámaras. Solo se sacó a las ratas de las cámaras experimentales durante una hora diaria (10:00 am) para registrar su peso, para pesar los bebederos y para limpiar las cámaras experimentales.

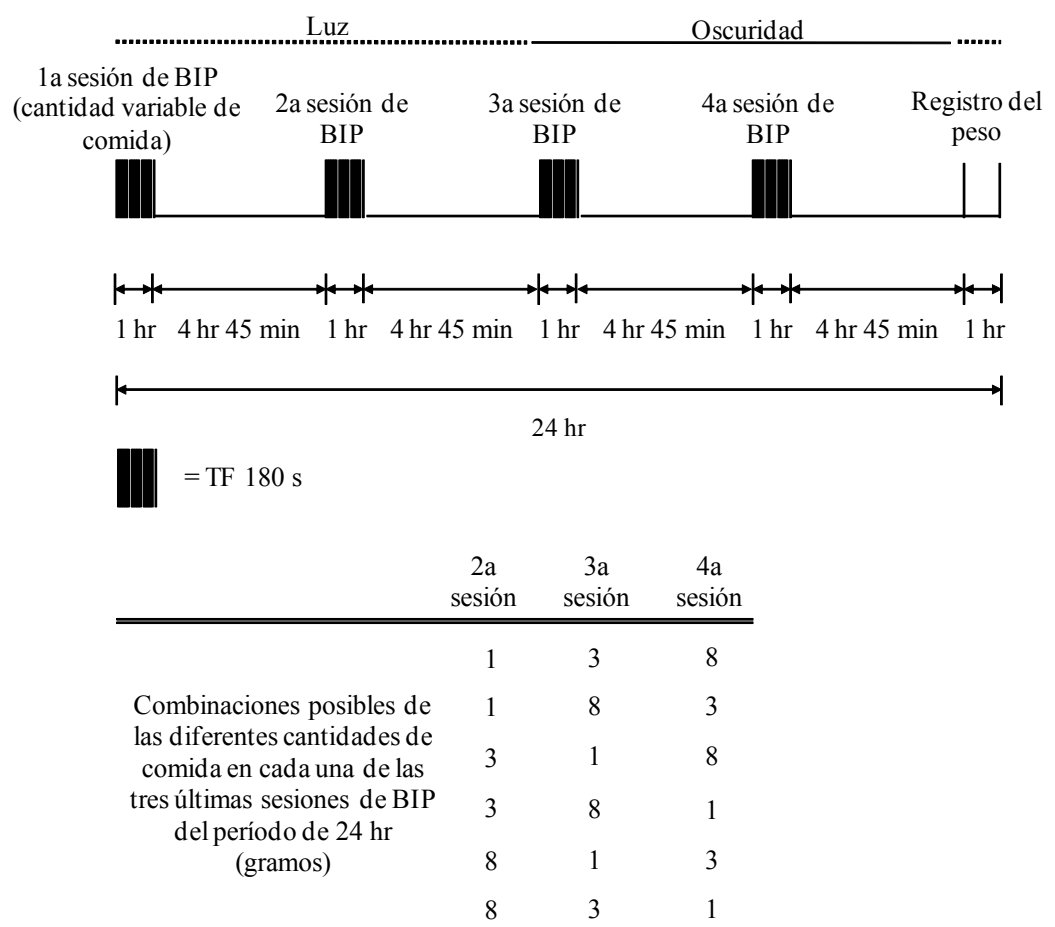

Figura 1. Esquema del procedimiento del Experimento 1. 
En la Figura 1 se muestra un esquema del procedimiento empleado en el presente experimento. Se programó un ciclo de luz-oscuridad de 11 hr 30 min dentro de las cámaras experimentales. Inmediatamente después de haber introducido a los sujetos en las cámaras experimentales, se programó una primera sesión de BIP, en la que se les entregaron a las ratas las bolas de comida necesarias para mantenerlas al $80 \%$ de su peso ad libitum conforme a un programa de tiempo fijo (TF) $180 \mathrm{~s}$ que estuvo en efecto durante una hora. Durante las siguientes $22 \mathrm{hr}$, cada 4 hr 45 min se programó una sesión de BIP que duraba una hora cada una (las 4 hr 45 min transcurrieron desde el final de una sesión de BIP al inicio de la siguiente sesión de BIP). Por lo tanto, además de la primera sesión del día en la que se les dio a las ratas la comida necesaria para mantenerlas al $80 \%$ de su peso ad libitum, se programaron tres sesiones de BIP adicionales. En cada una de las tres sesiones de BIP adicionales estuvo en efecto uno de tres programas de TF con los que se entregaron 2, 6 ó 16 bolas de comida cada $180 \mathrm{~s}$. De esta manera, después de que cada una de las tres sesiones finalizó, se completaron 1, 3 u 8 gramos de comida, respectivamente. $\mathrm{Si}$ bien las sesiones de BIP siempre se presentaron cada $4 \mathrm{hr} 45 \mathrm{~min}$, las tres sesiones (1, 3 u 8 gramos) se presentaron en un orden diferente cada día para evitar efectos de secuencia y para evitar que cada una de ellas ocurriera consistentemente durante el ciclo de luz o de oscuridad. En la parte inferior de la Figura 1 se muestran las seis posibles combinaciones del orden en el que se presentaron las sesiones a través del experimento. El experimento finalizó una vez que se completaron 30 días. Durante los 30 días se registraron los lengüetazos al tubo del bebedero continuamente.

El programa de entrega de comida de TF 180 s se eligió porque en estudios publicados sobre BIP, se mostró que éste programa es conducente a generar un consumo sustancial de agua durante las sesiones (e.g., Segal, Oden \& Deadwyler, 1965; Wetherington, 1979). Por ejemplo, Segal et al. entregaron comida a ratas conforme a un programa de TF y variaron la duración del intervalo entre comidas de 30 a 480 s. Encontraron que el consumo de agua fue una función de $U$ invertida de alargar la duración del intervalo entre comidas, y el máximo consumo de agua ocurrió con los intervalos entre comidas entre 120 y 240 s. Se eligieron las cantidades de comida de 2, 6 y16 bolas de comida cada $180 \mathrm{~s}$ debido a que en algunos estudios BIP en los que se determinaron los efectos de la cantidad de comida sobre el consumo de agua incluyen el rango que se exploró en el presente estudio (e. g., Lotter et al. 1973; Flory, 1971; Robinson \& Flory, 1989). Con base en una revisión sobre la literatura de BIP, en una gran cantidad de experimentos se expuso a las ratas a sesiones de BIP de 60 min, por lo tanto se eligió ésta duración para las sesiones, en las cuales estuvo vigente el programa de TF $180 \mathrm{s.}$

\section{Resultados}

Si bien durante tres sesiones de BIP siempre se entregaron 1, 3 u 8 gramos de comida, la cantidad de comida que se le entregó a los sujetos durante la primera sesión de 
BIP de cada período de 24 horas fue variable, ya que fue el complemento necesario para mantener a los sujetos al $80 \%$ de su peso ad libitum. La media de la cantidad de comida entregada durante la primera sesión de BIP de cada período de $24 \mathrm{hr}$ fue de $0.95,1.17$ y 1.10 para las Ratas 1,2 y 3 , respectivamente. Para los tres sujetos, la cantidad de comida entregada durante de la primera sesión de BIP únicamente varió entre 0.5 y 2.5 gramos de comida durante los 30 días del experimento.
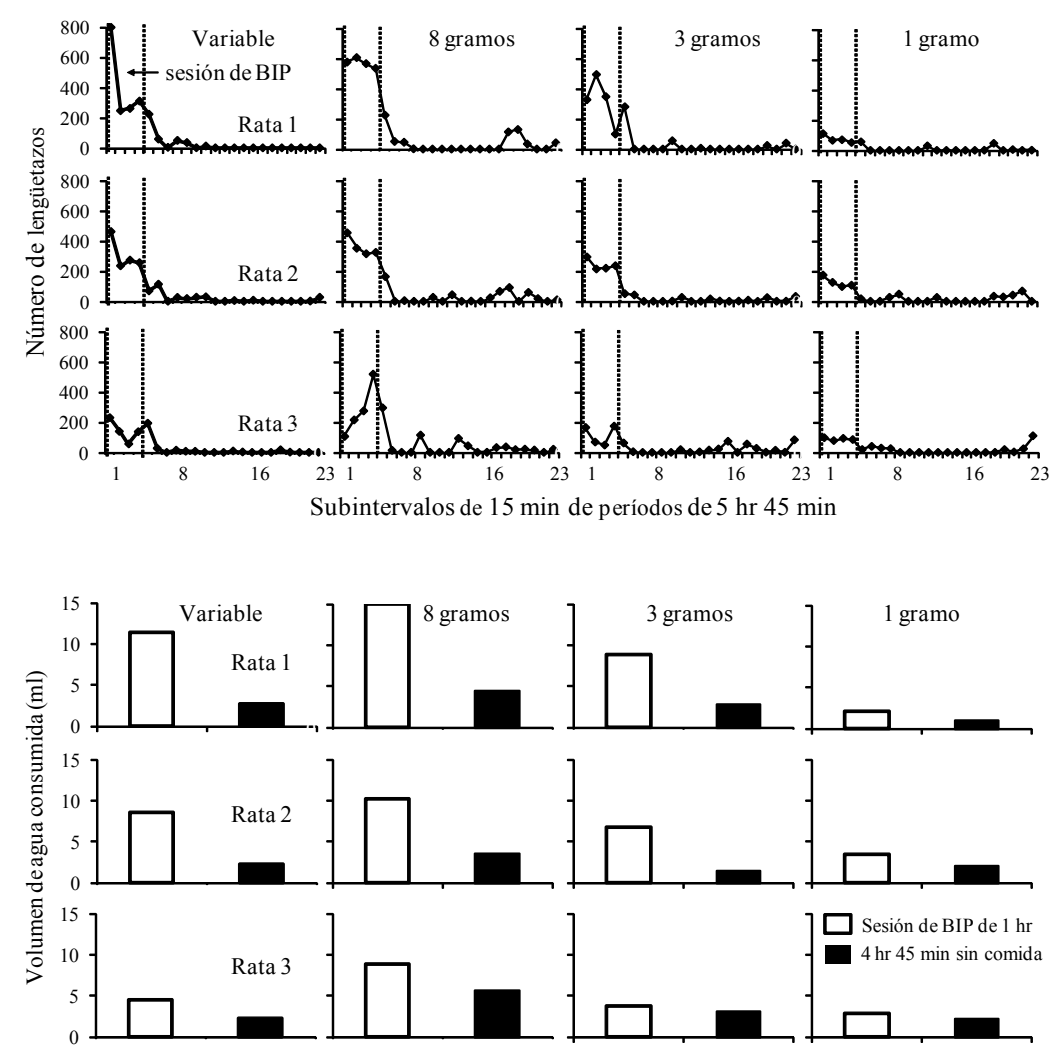

Figura 2. Panel superior: Distribución temporal de los lengüetazos al tubo en subintervalos de 15 min de cada una de las cuatro sesiones de BIP de una hora en las que se entregaron diferentes cantidades de comida y de cada uno de los cuatro períodos de 4 hr y 45 min posteriores a cada sesión. En el panel inferior se muestra el volumen de agua consumida durante cada una de las cuatro sesiones de BIP (barras blancas) y durante los cuatro períodos de $4 \mathrm{hr}$ y $45 \mathrm{~min}$ posteriores a cada sesión (barras negras). 
En el panel superior de la Figura 2 se presenta la distribución temporal de los lengüetazos por agua en subintervalos de 15 minutos de cada uno de los cuatro períodos de una hora en los que estuvieron vigentes las cuatro sesiones de BIP y durante las 4 hr y 45 min posteriores a cada sesión de BIP. Este dato se presenta para cada una de las tres ratas como la media del último bloque de cinco días. Las dos líneas punteadas en cada panel muestran el inicio y el final de la hora del día en la que estuvo en efecto cada sesión de BIP. El hallazgo a destacar es que para las tres ratas los lengüetazos por agua durante cada período de 23 horas estuvieron principalmente confinados dentro de las cuatro sesiones de BIP. Se observaron efectos sistemáticos de la cantidad de comida sobre el número de lengüetazos para las tres ratas. Se encontró que para todos los sujetos el número de lengüetazos fue mayor en las sesiones de BIP en las que se entregaron en total ocho gramos de comida (i.e., 16 bolas cada 180 s) que durante las sesiones en las que se entregaron tres o un gramo de comida (i.e., 6 ó 2 bolas cada 180 s, respectivamente). El menor número de lengüetazos ocurrió durante las sesiones de BIP en las que sólo se entregó un gramo de comida. Para las Ratas 1 y 2 el máximo número de lengüetazos ocurrió alrededor de los primeros 30 minutos de cada sesión y posteriormente el número de lengüetazos disminuyó gradualmente conforme transcurrió cada sesión. Para la Rata 3 la distribución temporal de los lengüetazos por agua a través de las sesiones de BIP no siguió un patrón sistemático. Cabe destacar que durante los 15 minutos posteriores al final de cada sesión de BIP ocurrieron varios lengüetazos por agua. El número de lengüetazos durante los 15 minutos posteriores a la sesión de BIP fue menor conforme se redujo la cantidad de comida entregada. Se observaron algunos lengüetazos por agua fuera de las cuatro sesiones de BIP para las tres ratas.

En el panel inferior de la Figura 2 se muestra el volumen de agua consumida durante cada una de las cuatro horas en las que estuvieron en efecto las diferentes sesiones de BIP (barras blancas) y durante cada uno de los cuatro períodos de $4 \mathrm{hr}$ y 45 min posteriores a cada sesión (barras negras). Disminuir la cantidad de comida entregada durante las sesiones de 8 a 1 gramo resultó en disminuciones progresivas en el volumen de agua consumida durante las sesiones para las tres ratas. Para las Ratas 1 y 3 disminuir la cantidad de comida durante las sesiones también tuvo efectos sistemáticos sobre el volumen de agua consumida durante los períodos de $4 \mathrm{hr}$ y 45 min que siguieron a cada una de las sesiones de BIP, en los cuales no se entregó comida. El volumen de agua consumida durante las sesiones conformó el $77.3 \%$, el $75.7 \%$ y el $60.3 \%$ de la ración diaria de agua de las Ratas 1, 2 y 3, respectivamente.

En la Figura 3 se muestra la distribución temporal de los lengüetazos en 180 subintervalos del intervalo entre comidas durante cada una de las cuatro sesiones de BIP que se presentaron durante el día. Este dato se presenta como la media del número total de lengüetazos para cada una de las tres ratas durante el último bloque de cinco días. Se encontró una función bitónica del lengüeteo por agua dentro del intervalo entre entregas de comida para los tres sujetos. La altura de cada función estuvo determinada por la cantidad de bolas de comida entregadas. A mayor canti- 
dad de bolas de comida entregadas por ocasión, el número de lengüetazos al tubo dentro del intervalo entre comidas fue mayor.

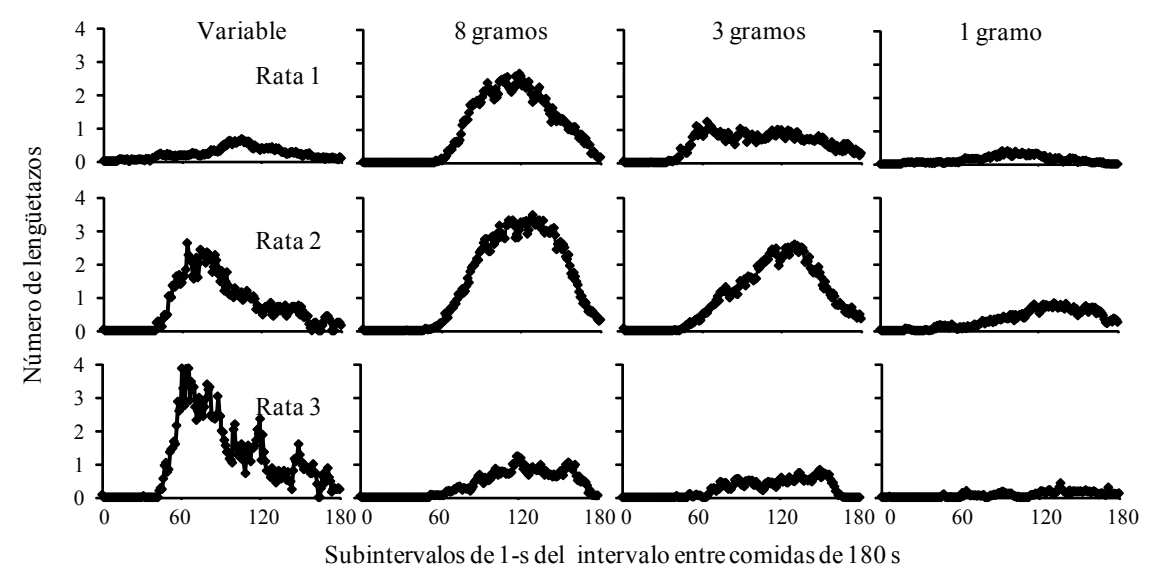

Figura 3. Distribución temporal de los lengüetazos en cada uno de los $180 \mathrm{~s}$ del intervalo entre comidas. Este dato se presenta para las tres ratas durante cada una de las cuatro sesiones de BIP en las que se entregaron diferentes cantidades de comida.

\section{Discusión}

Respecto a la distribución temporal del consumo de agua durante cada período de $24 \mathrm{hr}$, se encontró que los lengüetazos por agua se confinaron principalmente durante las cuatro sesiones de BIP. Este resultado muestra que a pesar de que las ratas tienen acceso continuo al agua, la mayor parte del consumo de agua ocurre en el momento del día en el que la comida se encuentra disponible y es congruente con los hallazgos de algunas investigaciones sobre el consumo de comida y agua en ratas. En los estudios en los que se analizó la distribución temporal del consumo diario de agua en ratas con acceso irrestricto a la comida y al agua se mostró que las ratas naturalmente alternan entre comer y beber y que estos episodios de comer y de beber se distribuyen a través de cada período de $24 \mathrm{hr}$ a intervalos regulares de tiempo (e.g., De Castro, 1989; Díaz \& Bruner, 2007; Fitzsimons \& Le Magnen, 1969). Adicionalmente, Fitzsimons y Le Magnen mostraron que al restringir el acceso a la comida a tan sólo dos horas del día, las ratas consumen alrededor del $70 \%$ de su ración diaria de agua en las dos horas en las que tenían la comida disponible. En el presente experimento, se replicó este hallazgo bajo las condiciones de BIP, esto es, con ratas privadas de comida expuestas a la entrega de comida a intervalos. Se encontró que bajo las condiciones de BIP, dos de las tres ratas bebieron poco más del $75 \%$ de su ración diaria de agua en presencia de la comida a intervalos. La tercera 
rata bebió alrededor del $60 \%$ de su ración diaria de agua durante las sesiones. Cabe destacar que al entregar la comida a intervalos dentro de las sesiones, se replicó la característica distribución temporal del consumo de agua de los experimentos de BIP, a la manera de una función bitónica (e. g., Staddon, 1977).

En los estudios sobre el patrón incondicionado de comer y beber en ratas, se reportó que la cantidad de comida y de agua que las ratas consumían durante el ciclo de oscuridad era mayor que durante el ciclo de luz (e. g., Díaz \& Bruner, 2007; Fitzsimons \& Le Magnen, 1969). Dado éste hallazgo, en el presente experimento se tomó la precaución de presentar las tres últimas sesiones de BIP (1, 3 y 8 gramos de comida) en un orden diferente en cada uno de los períodos de $23 \mathrm{hr}$, por lo que un tipo de sesión ocurrió tanto en el período de luz como en el período de oscuridad a través de los diferentes días del experimento. Independientemente de que las sesiones podían ocurrir en el período de luz o en el período de oscuridad, se encontró una clara consistencia entre la cantidad de comida entregada y la cantidad de agua que las ratas consumieron durante y después de las sesiones de BIP. A mayor cantidad de alimento disponible mayor fue el volumen de agua consumida. La consistencia entre la cantidad de comida entregada en cada sesión de BIP y el volumen de agua que las ratas consumieron dentro de las sesiones sugiere que la función de la comida a intervalos es la de restablecer el consumo de agua después de un período de restricción de comida.

Existen varios estudios de BIP en los que se determinaron los efectos de variar la cantidad de comida sobre el volumen de agua consumida durante las sesiones. En algunos de estos estudios se reportó que aumentar el número de bolas de comida resultó en un aumento en el volumen de agua consumida (e.g., Couch, 1974; Falk, 1969; Flory, 1971; Robinson \& Flory, 1989). No obstante, existen algunos experimentos en los cuales se encontró que conforme aumentó el número de bolas de comida entregadas, el volumen de agua consumida fue cada vez más bajo (e.g., Falk, 1967; Lotter et al. 1973). Por ejemplo, Lotter et al. (1973) reportaron que los aumentos en la cantidad de comida resultaron en disminuciones en el volumen de agua consumida durante la sesión. Expusieron a ratas a sesiones de BIP de una hora en las que entregaron comida conforme a un programa de IV 60 s. El comedero entregaba sólo una bola de comida de $45 \mathrm{mg}$ en cada operación. En un segundo experimento, mantuvieron constante la duración de las sesiones y el programa de IV 60 s, pero cada entrega de comida consistía de 12 bolas de alimento. Los autores reportaron que en el experimento en el que entregaron sólo una bola de alimento en cada operación del comedero, el volumen de agua consumida durante las sesiones fue mayor que el volumen de agua consumida cuando entregaron 12 bolas de alimento.

En contraste con los hallazgos de Lotter et al. (1973) Reid y Staddon (1982) encontraron que a mayor cantidad de comida, mayor fue el consumo de agua durante las sesiones. En algunos de sus experimentos, determinaron los efectos de entregar una ó cuatro bolas de comida conforme a un programa de TF. Los autores reportaron que el consumo de agua fue mayor durante las sesiones en las que entregaron cuatro 
bolas de comida. Un hallazgo a destacar es que encontraron que a mayor cantidad de comida entregada por ocasión, hubo un aumento en la duración de conductas relacionadas con la obtención de alimento (meter la cabeza en el recipiente de la comida).

El hallazgo de Reid y Staddon (1982) relativo a que aumentar la cantidad de comida resulta en aumentos en la duración de conductas relacionadas con la obtención del alimento permiten sugerir una posible explicación para los hallazgos mixtos en la literatura sobre BIP en los que se manipuló la cantidad de comida. Es posible que en los estudios en los que se encontró que aumentar la cantidad de comida resultó en una disminución del consumo de agua, dichos aumentos en la cantidad de comida también controlaron que las ratas pasaran un tiempo considerable de la sesión consumiendo la comida disponible, por lo que el consumo de agua ocurría una vez que se colocaba a los sujetos en sus cámaras experimentales. Los resultados del presente experimento también permiten sugerir ésta posible explicación. Se encontró que las ratas bebieron una cantidad sustancial de agua durante aproximadamente los quince minutos posteriores a las sesiones de BIP. Incluso, para dos de tres ratas, el volumen de agua consumida durante todo el período de 4 hr y 45 min en el que no se entregó comida aumentó conforme la cantidad de comida que se entregó durante las sesiones también aumentó. No es posible ofrecer una explicación convincente del origen de los hallazgos contradictorios entre los experimentos reportados en la literatura en los que se varió la cantidad de comida. Una dificultad para interpretar los hallazgos mixtos es que entre los experimentos, se manipularon simultáneamente diversas variables. Por ejemplo, se emplearon programas de entrega de comida de IF (e.g., Falk, 1967; Flory, 1971), de IV (e. g., Lotter et al. 1973) y de TF (e. g., Robinson \& Flory, 1989). Asímismo, se compararon cantidades diferentes de comida, las cuales variaron entre una y doce bolas de comida y la duración de las sesiones fue diferente entre los experimentos (ver Christian et al. 1977).

El presente experimento constituye una primera aproximación para explicar los efectos de la cantidad de comida sobre el consumo de agua en una situación de BIP, ya que ofrecen una perspectiva de lo que ocurre con el consumo de agua de las ratas durante y después de las sesiones en las que se manipula la cantidad de comida.

Experimento 2

Los hallazgos del Experimento 1 del presente estudio sugieren que la función de la comida durante las sesiones de BIP es la de restablecer el consumo de agua después de un período de tiempo en el que las ratas no reciben comida. Por lo tanto, se sugirió que el procedimiento de BIP era conducente a confinar al consumo diario de agua de las ratas al período de tiempo durante el cual está vigente la sesión de BIP. Surge la posibilidad de que el espaciamiento temporal de la comida es simplemente otra operación que garantiza que el consumo de agua ocurra dentro de las cámaras experimentales. Posiblemente, al dejar que transcurra un intervalo de tiempo entre 
entregas de comida sucesivas, el experimentador establece la oportunidad para que las ratas beban entre una bola de comida y la siguiente, por lo que observa la mayor parte del consumo diario de la rata durante la sesión.

Para determinar si el espaciamiento temporal de la comida sólo es una forma de garantizar que la mayor parte del consumo de agua ocurra durante las sesiones, el presente experimento fue una replicación sistemática del Experimento 1. Se emplearon las mismas ratas que en el Experimento 1, las cuales permanecieron continuamente dentro de las cámaras experimentales. En una primera fase se mantuvo constante la cantidad de comida entregada durante cada sesión de BIP (1, 3 u 8 gramos), sin embargo se les entregaron a las ratas todas las bolas de comida juntas al inicio de cada sesión. En la segunda fase del experimento, se entregaron los 12 gramos de bolas de comida al inicio de cada período de $24 \mathrm{hr}$. Si el espaciamiento temporal de la comida sólo es una operación que garantiza que el beber diario ocurra principalmente durante las sesiones, la predicción para el presente experimento fue las variaciones en la distribución temporal de la entrega de la comida únicamente tendrían efectos sobre la distribución temporal del consumo de agua, pero no sobre el volumen de agua que consumen las ratas en cada período de $24 \mathrm{hr}$. El propósito del Experimento 2 fue determinar los efectos de la distribución temporal de la entrega de la comida sobre el consumo de agua durante períodos de 24 horas.

\section{Método}

\section{Sujetos}

Se usaron las mismas tres ratas que en el Experimento 1. Las ratas permanecieron privadas de comida al $80 \%$ de su peso ad libitum y tuvieron acceso libre al agua durante todo el experimento.

\section{Aparatos}

Los mismos que se emplearon en el Experimento 1.

\section{Procedimiento}

El Experimento 2 consistió de dos fases consecutivas de 30 días cada una. Al igual que en el Experimento 1, durante las dos fases del experimento las ratas permanecieron en la cámara experimental en la cual el tubo del bebedero con agua estuvo disponible continuamente. Durante cada día se programó un ciclo de luz-oscuridad de $11.5 \mathrm{hr}$. Solo se sacó a las ratas de las cámaras experimentales durante una hora diaria $(11: 00 \mathrm{am})$ para registrar su peso, para pesar los bebederos y para limpiar las cámaras experimentales.

Las dos fases del Experimento 2 únicamente difirieron entre sí en la distribución temporal de la entrega de la comida. En la Figura 4 se muestra un esquema del procedimiento empleado en cada una de las dos fases. 


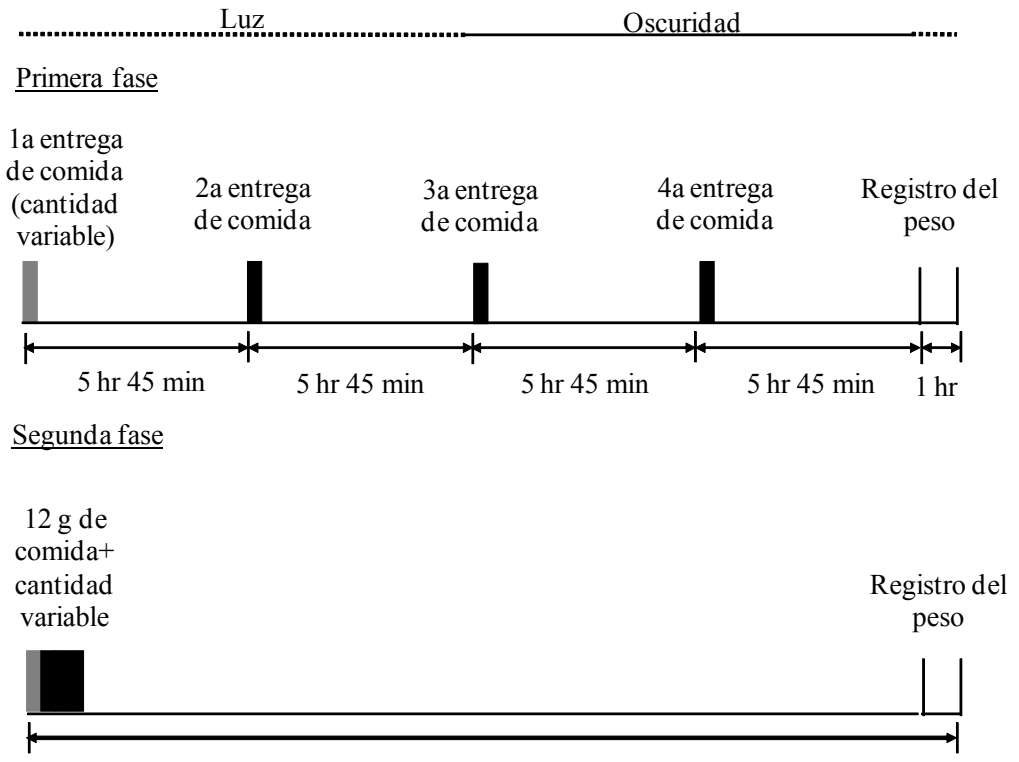

Figura 4. Esquema del procedimiento del Experimento 2.

Fase 1: Entrega de comida junta cada 5 hr y 45 min

Inmediatamente después de haber finalizado el Experimento 1 se colocó a los sujetos nuevamente en las cámaras experimentales e inició la primera fase del Experimento 2. Se programaron cuatro entregas de comida en rápida sucesión dentro de cada período observación de $23 \mathrm{hr}$. El intervalo entre una bola de comida y la siguiente fue de $0.5 \mathrm{~s}$. En la primera entrega de comida, se le dio a las ratas la comida necesaria para mantenerlas al $80 \%$ de su peso ad libitum. Al igual que en el Experimento 1, durante las siguientes $22 \mathrm{hr}$ se programó un período de entrega de comida cada 5 hr 45 min. En cada una de las tres entregas de comida, se entregaron 1, 3 u 8 g de comida, al igual que en el Experimento 1. El orden de los períodos de entrega de comida (1, 3 u 8 gramos) fue diferente cada día para evitar que cada entrega de comida $(1,3$ u 8 g) ocurriera consistentemente en el ciclo de luz o en el ciclo de oscuridad.

Fase 2: Entrega de comida junta al inicio de cada período de $24 \mathrm{hr}$ En la segunda fase del experimento se determinaron los efectos de presentar todas las bolas de comida juntas al inicio del día sobre el consumo de agua durante el período de $23 \mathrm{hr}$. Inmediatamente después de haber colocado a los sujetos dentro de las cámaras experimentales (10:00 am) se entregaron los 12 gr de comida más el número de bolas necesarias para mantener a las ratas al $80 \%$ de su peso ad libitum en rápida sucesión (cada 0.5 s.). 
Beber inducido por el programa

Resultados

Al igual que en el Experimento 1 en el que se entregó la comida conforme a las "sesiones" de BIP, la cantidad total de comida entregada diariamente varió entre 12.5 y 14.5 gramos de comida, por lo que durante la segunda fase se siguió entregando únicamente entre 0.5 y 2.5 gramos de comida como complemento de los 12 gramos programados para mantener a las ratas al $80 \%$ de su peso ad libitum.

La hipótesis que se planteó en el presente experimento fue que el espaciamiento temporal de la entrega de la comida no resulta en un aumento en el volumen de agua que consumen las ratas, sino que es una operación conducente a que la mayor parte del consumo diario de las ratas ocurra durante las sesiones de BIP. Por lo tanto, la variable dependiente más importante del presente experimento fue el volumen de agua consumida durante las dos fases del presente experimento en comparación con el volumen de agua consumida durante el Experimento 1, en el que se entregó la comida conforme a un TF 180 s. En la Figura 5 se muestra el volumen de agua consumida durante cada período de 23 horas en cada una de las dos fases del presente experimento. En la Figura 5 también se muestra el volumen de agua consumida durante los días en los que se le entregó a los sujetos la comida conforme al TF $180 \mathrm{~s}$ (Experimento 1).

Se compararon las medias del volumen de agua consumida en los 30 días de cada una de las dos fases del presente experimento y en cada uno de los días del Experimento 2 usando pruebas $t$ para muestras apareadas. Se corrigió el nivel alfa para tres repeticiones de la prueba $t$ con la misma muestra (alfa $=.05 / 3=0.016$ ). Se encontró que el volumen de agua consumida no fue diferente durante la fase en la que estuvieron en efecto las cuatro sesiones de BIP $(M=42.63, D E=4.45)$ que durante la fase en la que se entregó a las ratas toda la comida junta al inicio del día $(M=35.18, D E=0.84), t(2)=2.48, p=0.13$. El volumen de agua consumida durante la fase en la que se entregó la comida conforme a las sesiones de BIP tampoco fue diferente del volumen de agua consumida en la fase en la que se entregó a las ratas la comida junta cada 5 hr 45 min $(M=34.90, D E=1.44), t(2)=3.11, p=0.90$. Tampoco se encontraron diferencias en el volumen de agua consumida durante la fase en la que se entregó toda la comida al inicio del día y en la fase en la que se entregó comida junta cada 5 hr 45 min, $t(2)=0.33, p=0.77$.

En resumen, variar la distribución temporal de la entrega de la comida no tuvo efectos sobre el volumen de agua que los sujetos consumieron durante cada período de 23 horas. El volumen de agua consumida concurrente con la entrega de comida a intervalos de tiempo en el Experimento 1 no fue diferente del consumo de agua durante los días en los que se les presentó a los sujetos la comida en rápida sucesión al inicio de las sesiones e incluso cuando se les entregó toda la comida al inicio de cada período de 24 horas (Experimento 2). 


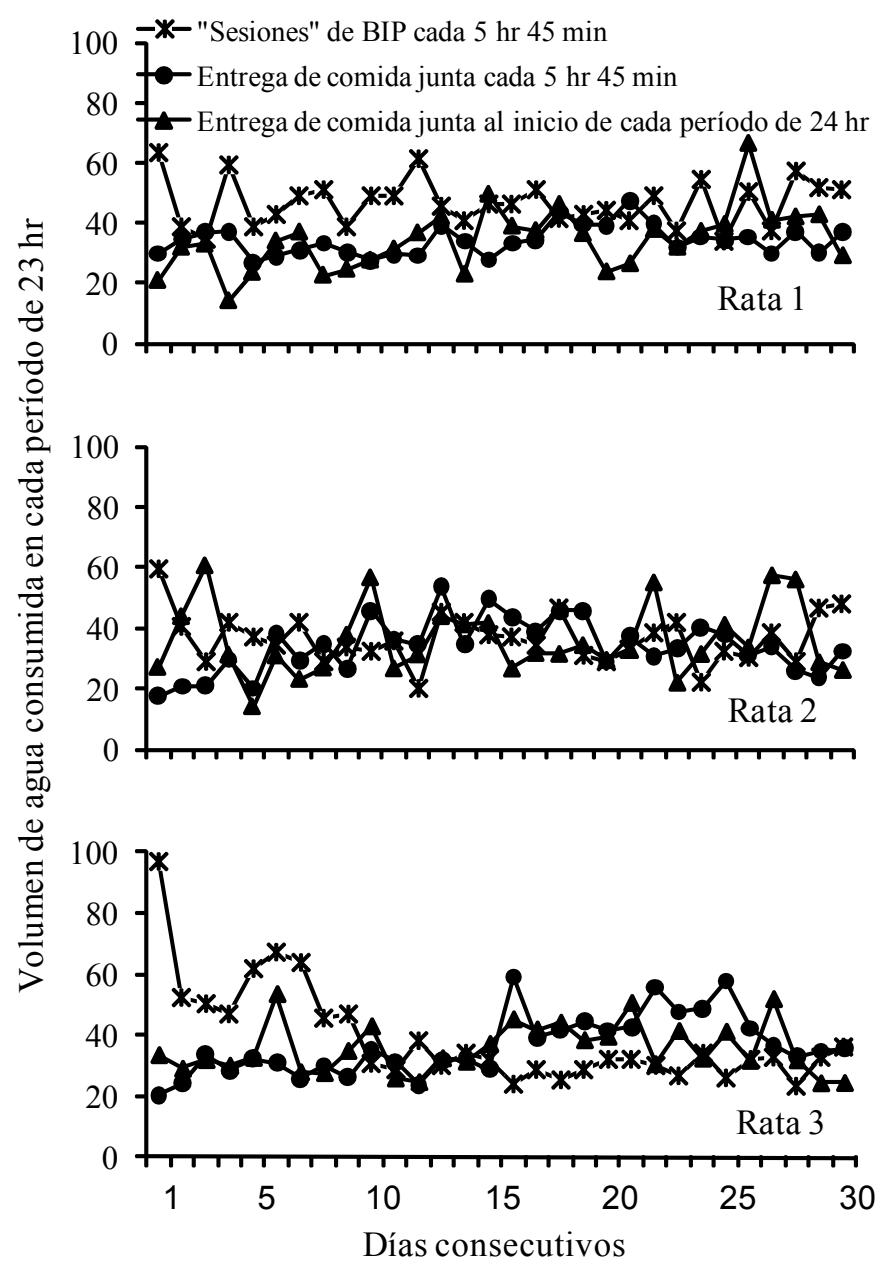

Figura 5. Volumen de agua consumida durante cada uno de los 30 días de la fase en la que se entregó la comida junta cada 5 hr y 45 min y durante los 30 días de la fase en la que se entregó toda la comida junta al inicio del día. En la figura también se presenta el volumen de agua consumida durante cada uno de los 30 días del Experimento 1. 

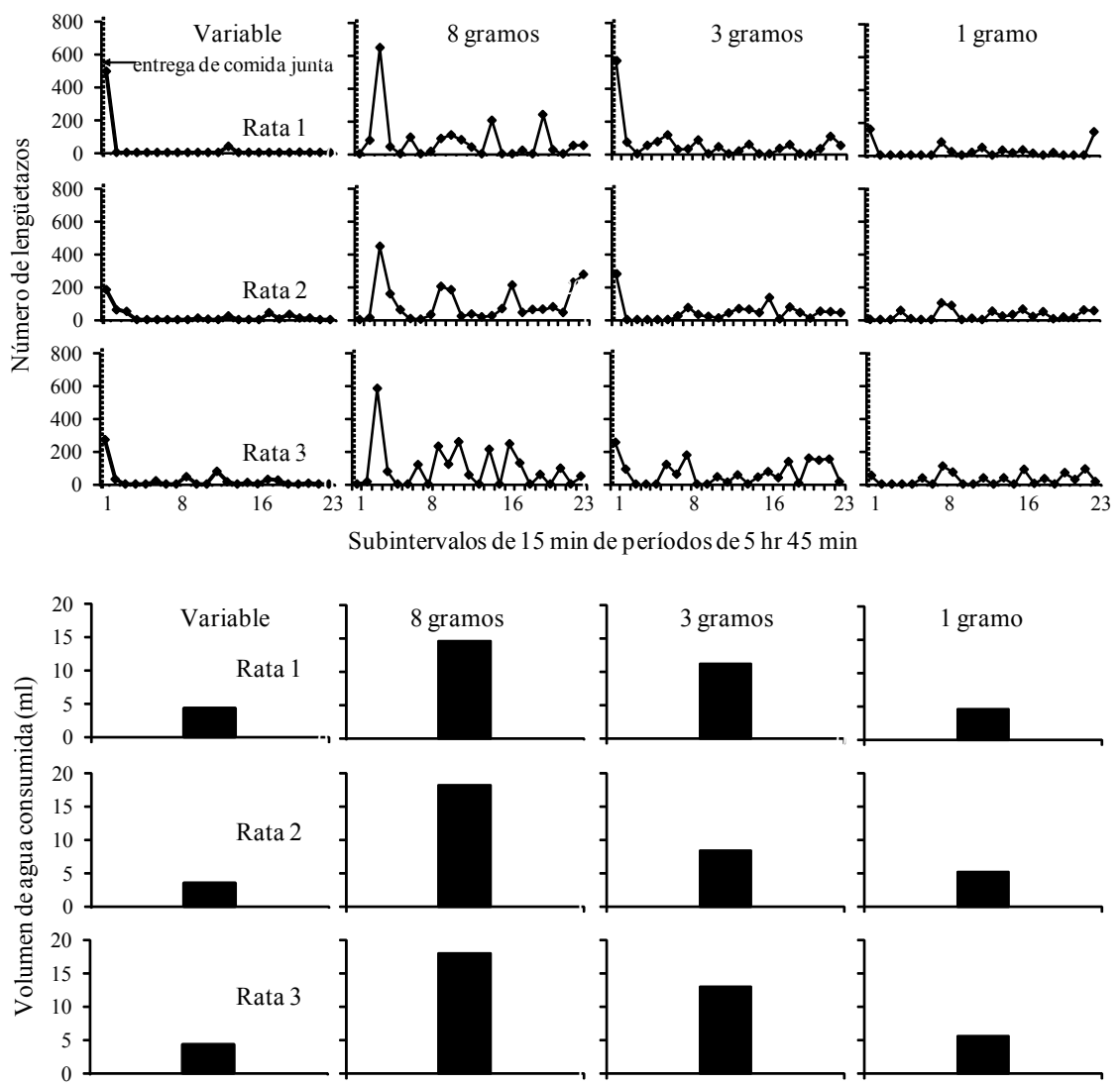

Figura 6. Panel superior: Distribución temporal de los lengüetazos por agua durante la primera fase del Experimento 2. La distribución temporal de los lengüetazos se presenta en subintervalos de $15 \mathrm{~min}$ de cada uno de los cuatro períodos de $5 \mathrm{hr}$ y 45 min que transcurrieron entre las entregas de comida en rápida sucesión. En el panel inferior se muestra el volumen de agua consumida durante cada uno de los cuatro períodos de $5 \mathrm{hr}$ y $45 \mathrm{~min}$.

En el panel superior de la Figura 6 se muestra la distribución temporal del número de lengüetazos al tubo del bebedero durante la fase en la que se entregó la comida cada 5 hr y 45 min. La distribución temporal de los lengüetazos se presenta en subintervalos de 15 minutos de cada período de $5 \mathrm{hr} 45$ min para cada una de las tres ratas como la media del último bloque de cinco días. Se encontró que entregar todas las bolas de comida juntas al inicio de cada "sesión" de BIP controló que los 
lengüetazos por agua se distribuyeran a través de todo el período de 23 horas. Cuando se entregaron 8 gramos de comida se observó que hubo un aumento abrupto en el número de lengüetazos durante el tercer subintervalo de cada período de $5 \mathrm{hr}$ y 45 min para las tres ratas. Cuando se entregaron 3 gramos de comida, se observó que el máximo número de lengüetazos ocurrió en contigüidad con la entrega de la comida (en el primer subintervalo de cada período de $5 \mathrm{hr}$ y $45 \mathrm{~min}$ ). Cuando se entregó un gramo de comida, el consumo de agua se a niveles cercanos a cero durante cada período de $5 \mathrm{hr}$ y $45 \mathrm{~min}$.

En el panel inferior de la Figura 6 se muestra el volumen de agua consumida durante cada período de 5 hr y 45 min en función de la cantidad de comida que se entregó en cada uno de ellos. Se encontró que para los tres sujetos, a menor cantidad de comida entregada, el volumen de agua consumida durante cada período de $5 \mathrm{hr}$ y 45 min fue también menor. El volumen de agua consumida durante el período en el que se entregó una cantidad variable de comida fue similar al volumen de agua consumida durante el período en el que se entregó un gramo.

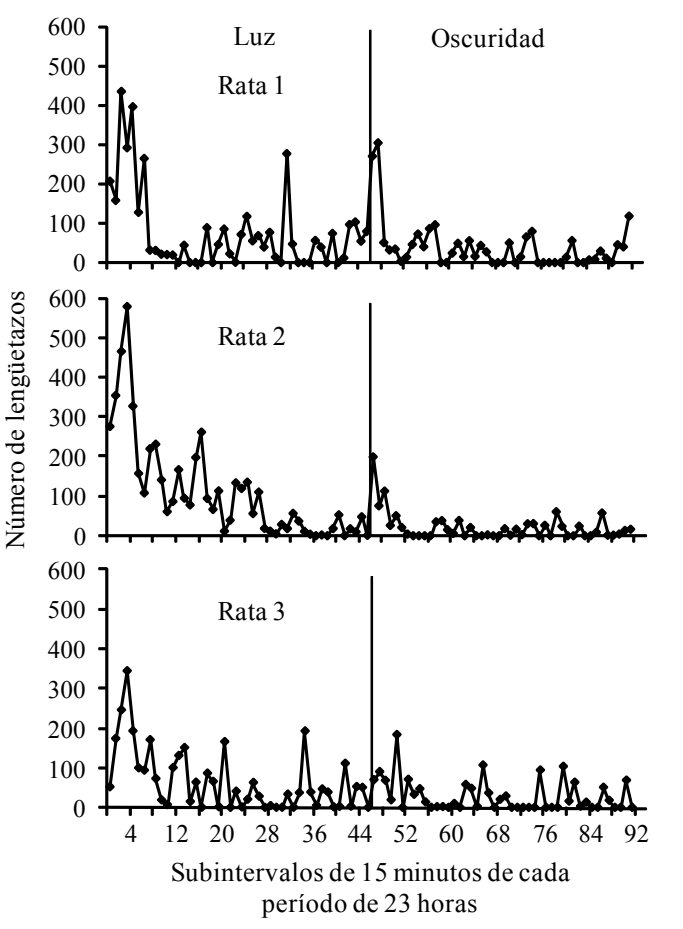

Figura 7. Distribución temporal del número de lengüetazos al tubo del bebedero en subintervalos de 15 minutos de cada período de $23 \mathrm{hr}$ de la segunda fase del Experimento 2. 
En la Figura 7 se presenta la distribución temporal del número de lengüetazos al tubo del bebedero en subintervalos de 15 minutos de cada período de $23 \mathrm{hr}$ de la segunda fase del experimento, en la cual se entregaron todas las bolas de comida juntas al inicio de cada período de $24 \mathrm{hr}$. Este dato se presenta para cada una de las tres ratas como la media del último bloque de cinco días de la segunda fase del experimento. La línea continua indica el inicio del ciclo de oscuridad. El máximo número de lengüetazos durante el período de $23 \mathrm{hr}$ ocurrió dentro de la primera hora del día para las tres ratas, esto es, en cercanía temporal con la entrega de la comida. Se observaron varios lengüetazos al tubo distribuidos en todo el período de $23 \mathrm{hr}$.

\section{Discusión}

En el Experimento 2, no se observaron diferencias entre el volumen de agua consumida durante la fase en la que se entregó la comida junta cada 5 hr y 45 min y durante la fase en la que se entregó toda la comida junta al inicio del día. No obstante, el hallazgo más importante relativo a los efectos de la distribución temporal de la comida sobre el consumo de agua surge al comparar el consumo global de agua en los Experimentos 1 y 2 (ver Figura 5). Los datos del volumen de agua consumida durante ambos experimentos sugieren que el espaciamiento temporal de la comida no resulta en un consumo excesivo de agua, sino que simplemente establece la ocasión para que las ratas beban entre una entrega de comida y la siguiente. Se mostró que al entregar comida a intervalos de tiempo, la mayor parte del consumo de agua ocurrió dentro de las sesiones de BIP, y presentar la comida junta resultó en que las ratas bebieran agua durante todo el período de 24 horas. La distribución de la entrega de comida en los estudios de BIP tiene efectos sistemáticos sobre la distribución temporal del consumo de agua pero no sobre el volumen de agua que consumen los sujetos.

Uno de los criterios que se siguió en la literatura para afirmar que el BIP era excesivo se basó en la comparación entre el volumen de agua que los sujetos consumían al presentarles comida a intervalos de tiempo con el volumen de agua que consumían al presentarles todas las bolas de comida simultáneamente. En varios estudios se mostró que el consumo de agua era considerablemente mayor si se distribuía a lo largo de la sesión una cantidad determinada de bolas de comida que si se colocaba la misma cantidad de comida en un recipiente desde el inicio de la sesión (e.g., Brush \& Schaeffer, 1974; Christian, 1975; King et al. 1972). Los datos del presente experimento sugieren que en los estudios de BIP entregar la comida a intervalos de tiempo pudo haber controlado que las ratas bebieran agua exclusivamente durante la sesión experimental. En contraste, cuando se presentaron las bolas de comida juntas desde el inicio de la sesión, posiblemente las ratas pasaban un período de tiempo considerable consumiendo la comida y por lo tanto el consumo de agua pudo haber ocurrido después de las sesiones, una vez que los sujetos se encontraban en sus cajas habitación. 
En la fase del presente experimento en la que se entregaron las bolas de comida juntas al inicio de cada período de 5 hr y 45 min se encontró que el momento en el que las ratas consumieron la mayor parte de su ración de agua dependió de la cantidad de comida entregada. Cuando se entregaron 8 gramos de comida la mayor parte del consumo de agua del período de $5 \mathrm{hr}$ y 45 min ocurrió aproximadamente 30 min después de haber entregado la comida. En contraste, cuando se entregaron 3 gramos de comida, el máximo número de lengüetazos por agua ocurrió dentro de los primeros 15 minutos de cada período de 5 hr y 45 min. Estas diferencias en la distribución temporal del consumo de agua posiblemente se deben a que los sujetos permanecen consumiendo las bolas de comida disponibles en el recipiente y posteriormente beben el agua. Por lo tanto, a mayor cantidad de comida entregada mayor es el tiempo que transcurre entre la entrega de comida y el momento en el que las ratas comienzan a beber.

En la fase en la que se entregó comida en rápida sucesión al inicio de las sesiones se encontró que aumentar la cantidad de comida resultó en aumentos en el volumen de agua consumida en cada período de $5 \mathrm{hr}$ y $45 \mathrm{~min}$. Este hallazgo es congruente con el Experimento 1 y muestra que el volumen de agua consumida es proporcional a la cantidad de comida entregada, pero que cada episodio de beber contribuye a un consumo de agua relativamente constante en cada período de $24 \mathrm{hr}$.

\section{Discusión general}

Los hallazgos de los dos experimentos del presente estudio sugieren que el procedimiento de BIP es un conjunto de diferentes operaciones que controlan que las ratas beban agua consistentemente en un período del día. Dada la interacción natural entre las conductas de comer y de beber de las ratas, bajo los procedimientos de BIP la restricción de comida resulta en que las ratas beban agua infrecuentemente en sus cajas habitación. Restringir la comida en las cajas habitación es una operación que garantiza que las ratas consuman las bolas de comida durante las sesiones, por lo tanto, beban la mayor parte de su ración diaria de agua dentro de las cámaras experimentales. El espaciamiento temporal de la comida durante las sesiones de BIP resulta en que el consumo de agua ocurra principalmente durante las sesiones y no una vez que se coloca a las ratas en sus cajas habitación. Los datos del presente estudio sugieren que el espaciamiento temporal de la comida no es una variable que determina el volumen de agua que consumen las ratas, sino una variable que determina la distribución temporal del beber. Al entregar la comida a intervalos dentro de las "sesiones" se replicó la característica distribución temporal del consumo de agua dentro del intervalo entre comidas que se reportó en los experimentos de BIP, a la manera de una función bitónica (Staddon, 1977). Surge la posibilidad de que la función bitónica, la cual se ha considerado como una característica distintiva del BIP se deba a que la entrega de comida a intervalos acentúa la alternación natural entre el consumo de comida y de agua de las ratas. 
Es necesario reconocer que no es posible todavía hacer conclusiones finales sobre el origen de la naturaleza excesiva del consumo de agua en los procedimientos de BIP. El presente estudio constituye una aproximación para explicar el consumo sustancial de agua durante las sesiones en términos de privaciones indirectas y de la interacción entre el consumo de comida y de agua una vez que se restablece la comida durante las sesiones experimentales. Los hallazgos del Experimento 1, en el que se presentó la comida a intervalos de tiempo, llevaron a conducir el segundo experimento reportado en el presente estudio, en el que se presentó la comida toda junta cada 5 hr y 45 min y posteriormente al inicio de cada período de 23 horas. Por lo tanto, no es posible descartar que la secuencia de las manipulaciones de la distribución temporal de la comida determinó que el consumo de agua se mantuviera relativamente constante durante ambos experimentos. No obstante, hasta donde los autores saben, no hay razones en la literatura que lleven a la conclusión de que la secuencia de las manipulaciones de variar la distribución temporal de la comida determinó el hallazgo del presente estudio relativo a la constancia del consumo de agua. En futuras investigaciones podría replicarse sistemáticamente el presente estudio, de tal forma que se contrabalanceara el orden de presentación de las diferentes condiciones en las que se manipuló la distribución temporal de la comida.

La excesividad del consumo de agua se ha considerado como una característica distintiva del BIP en términos de una conducta inducida por el programa (e. g., Falk, 1971). Por lo tanto, la explicación del BIP conforme a los principios del análisis conductual ha dependido en parte de establecer las razones por las cuales las ratas beben grandes cantidades de agua durante las sesiones (cf. Wetherington, 1982). El supuesto detrás del argumento de que las ratas bebían cantidades sustanciales de agua fue que dado que bajo el procedimiento de BIP las ratas tenían una fuente de agua disponible continuamente, no existía una razón aparente para que las ratas bebieran agua consistentemente durante las sesiones, ya que no estaban privadas de agua (e. g... Falk, 1971). No obstante, los hallazgos del presente estudio mostraron que en la ausencia de comida, las ratas beben agua infrecuentemente, aún cuando tienen la botella con agua disponible continuamente. La privación de comida, su posterior restablecimiento en las cámaras experimentales e incluso el espaciamiento temporal de la comida son operaciones que al combinarlas resultan confiablemente en que el consumo diario de agua de las ratas se confine a las sesiones de BIP.

Desde la década de los años sesenta varios investigadores han intentado explicar la ocurrencia del BIP. Surgieron varias hipótesis para intentar explicar la naturaleza del consumo de agua, pero ninguna de ellas fue consistente con los principios del análisis conductual. Asimismo, debido a que se consideró a la comida como el único reforzador presente durante las sesiones e incluso a que el consumo de agua parecía ser "excesivo", en la literatura predominaron los fracasos en explicar al BIP en términos de conducta operante o respondiente. La falta de generalidad o bien de verificación empírica de las explicaciones sobre la ocurrencia del BIP y la imposibilidad de controlarlo mediante alguno de los dos tipos de condicionamiento, 
determinaron su permanencia como una "tercera categoría conductual" (ver Wetherington, 1982, para una revisión).

Existen demostraciones de que el BIP se trata de una conducta operante reforzada directamente por el agua (e. g., Bruner \& Ávila, 2002; Roca \& Bruner, 2003; Ruiz \& Bruner, 2008). Al disociar la conducta de beber en una respuesta instrumental y su consecuencia, i.e., la entrega de gotas de agua, fue posible replicar diferentes fenómenos del condicionamiento operante bajo las condiciones de BIP. En estos estudios se mostró que el agua refuerza directamente a las conductas que la producen, como las presiones a una palanca o los lengüetazos a un tubo. Por lo tanto, el siguiente paso para la explicación integral del BIP como un caso de conducta operante era clarificar por qué el agua adquiría propiedades reforzantes aún en la ausencia de una privación explícita de agua bajo el procedimiento de BIP y esclarecer su carácter excesivo. La privación de comida y la entrega de comida a intervalos en las cámaras experimentales son operaciones de establecimiento que dotan al agua de propiedades reforzantes durante las sesiones.

El presente estudio constituye una aproximación para explicar el BIP en términos de la interacción entre el agua y la comida. La disponibilidad de la comida resulta en un aumento en el valor reforzante del agua, y en la ausencia de comida las ratas dejan de beber. Es posible explicar el BIP en términos de la complementariedad de ambos reforzadores. En términos de la economía conductual, la interacción entre dos reforzadores se determina por el grado en el que los reforzadores son sustitutos o complementarios (ver Green \& Freed, 1993 para una revisión). Si aumentar la disponibilidad de un estímulo resulta en una disminución en el valor reforzante del segundo estímulo, ambos reforzadores son sustitutos. Los reforzadores sustitutos típicamente son estímulos que comparten ciertas características. Por ejemplo, tanto el café como el té contienen cafeína. El té podría ser un sustituto del café si los aumentos en el precio del café (y por lo tanto en disminuciones en el consumo de café) resultaran en un aumento en el consumo del té (Madden, Smethells, Ewan \& Hursh, 2007). En otras palabras, el café y el té son reforzadores sustitutos debido a que la disponibilidad del café resulta en una disminución del valor reforzante del té.

Los reforzadores complementarios están en el extremo opuesto a los reforzadores sustitutos dentro de un continuo conformado por las posibles interacciones entre los reforzadores. Dos reforzadores son complementarios cuando la disponibilidad del primer estímulo aumenta el valor reforzante del segundo estímulo. En términos de la economía conductual, los reforzadores que guardan una perfecta relación de complementariedad se consumen juntos, a una proporción constante, independientemente del aumento en el precio de uno de ellos. En varios experimentos, se ha mostrado la relación de complementariedad del agua y de la comida (e. g., Green \& Rachlin, 1991; Hursh, 1978; Madden et al. 2007). En estos estudios se mostró que el consumo del agua disminuye conforme el consumo de comida disminuye debido al aumento en el precio de la comida (respuestas requeridas para obtener la comida). Complementariamente, las disminuciones en el precio de la comida (y por lo tanto los 
Beber inducido por el programa

aumentos en el consumo de comida) resultan en aumentos en el consumo del agua.

La relación de complementariedad del agua y de la comida explica, en parte, por qué en los procedimientos de BIP la ausencia de comida resulta en una disminución en el consumo de agua y una vez que se entrega la comida durante las sesiones el consumo de agua se restablece. Aún más, explica por qué los aumentos en la cantidad de comida entregada resultan en aumentos proporcionales en la cantidad de agua que beben las ratas dentro y fuera de las sesiones de BIP.

Dado que el BIP puede explicarse con base en un conjunto de principios bien establecidos dentro del análisis experimental de la conducta, su permanencia como un fenómeno peculiar parece ser injustificada.

\section{Referencias}

Allen, J. D., Porter, J. H., \& Arazie, R. (1975). Schedule-induced drinking as a function of percentage reinforcement. Journal of the Experimental Analysis of Behavior, 23, 223-232, available via: http://dx.doi.org/10.1901/jeab.1975.23-223

Bolles, R. C. (1961). The interaction of hunger and thirst in the rat. Journal of Comparative and Physiological Psychology, 54, 580-584, available via: http://dx.doi. org $/ 10.1037 / \mathrm{h} 0044595$

Bruner, C. A., \& Ávila, R. (2002). Adquisición y mantenimiento del palanqueo en ratas sin privación explícita del reforzador. Revista Mexicana de Análisis de la Conducta, 28, 107-130.

Brush, M. E., \& Schaeffer, R. W. (1974). Effects of water deprivation on scheduleinduced polydipsia. Bulletin of the Psychonomic Society, 4, 69-72.

Christian, W. P. (1975). Effects of the quality of dry food reinforcement on the rat's instrumental licking and drinking behavior. The Psychological Record, 25, 237-242.

Christian, W. P., Schaeffer, R. W., \& King, G. D. (1977). Schedule-induced Behavior. Montreal: Eden.

Clark, F. C. (1962). Some observations on the adventitious reinforcement of drinking under food reinforcement. Journal of the Experimental Analysis of Behavior, 5, 61-63, available via: http://dx.doi.org/10.1901/jeab.1962.5-61

Couch, J. V. (1974). Reinforcement magnitude and schedule-induced polydipsia: A reexamination. Psychological Record, 24, 559-562.

De Castro, J. M. (1989). The interactions of fluid and food intake in the spontaneous feeding and drinking patterns of rats. Physiology and Behavior, 45, 861-870.

Díaz, F. J., \& Bruner, C. A. (2007). Comer y beber en ratas con libre acceso a la comida y al agua. Acta Comportamentalia, 15, 111-130.

Falk, J. L. (1961). Production of polydipsia in normal rats by an intermittent food schedule. Science, 133, 195-196.

Falk, J. L. (1964). Studies on schedule-induced polydipsia. En M. J. Wayner (Ed.), Thirst: First International Symposium on Thirst in the Regulation of Body Water (pp. 95-116). Nueva York: Pergamon Press. 
Falk, J. L. (1966b). Schedule-induced Polydipsia as a function of fixed-interval length. Journal of the Experimental Analysis of Behavior, 9, 37-39, available via: http://dx.doi.org/10.1901/jeab.1966.9-37

Falk, J. L. (1967). Control of schedule-induced Polydipsia: Type, size, and spacing of meals. Journal of the Experimental Analysis of Behavior, 10, 199-206, available via: http://dx.doi.org/10.1901/jeab.1967.10-199

Falk, J. L. (1969). Conditions producing psychogenic polydipsia in animals. Annals of the New York Academy of Sciences, 157, 569-593, available via: http://dx.doi. org/10.1111/j.1749-6632.1969.tb12908.x

Falk, J. L. (1971). The nature and determinants of adjunctive behavior. Physiology and Behavior, 6, 577-588, available via: http://dx.doi.org/10.1016/0031-9384(71)90209-5

Falk, J. L. (1977). The origin and functions of adjunctive behavior. Animal Learning and Behavior, 5, 325-335, available via: http://dx.doi.org/10.3758/BF03209574

Fitzsimons, T. J., \& Le Magnen, J. (1969). Eating as a regulatory control of drinking in the rat. Journal of Comparative and Physiological Psychology, 67, 273-283, available via: http://dx.doi.org/10.1037/h0026772

Flory, R. K. (1971). The control of schedule-induced polydipsia: Frequency and magnitude of reinforcement. Learning and Motivation, 2, 215-227, available via: http://dx.doi.org/10.1016/0023-9690(71)90022-1

Freed, E., \& Hymowitz, N. (1972). Effects of schedule, percent body weight, and magnitude of reinforcer on the acquisition of schedule-induced polydipsia. Psychological Reports, 31, 95-101.

Green, L., \& Freed, D. E. (1993). The substitutability of reinforcers. Journal of the Experimental Analysis of Behavior, 60, 141-158, available via: http://dx.doi. org/10.1901/jeab.1993.60-141

Green, L., \& Rachlin, H. (1991). Economic substitutability of electrical brain stimulation, food, and water. Journal of the Experimental Analysis of Behavior, 55, 133-143, available via: http://dx.doi.org/10.1901/jeab.1991.55-133

Hursh, S. R. (1978). The economics of daily consumption controlling food- and water reinforced responding. Journal of the Experimental Analysis of Behavior, 29, 475-491, available via: http://dx.doi.org/10.1901/jeab.1978.29-475

King, G. D., Mc Gill, D., Pierson, S. C., \& Schaeffer, R. W. (1972). Schedule-induced alcohol and water intakes in rats on a FFI 60 sec. schedule. Psychological Reports, 30, 291-296.

López-Espinoza, A., \& Martínez, H. (2005). Efectos de intervalos variables entre períodos de privación sobre el consumo post-privación de agua y comida en ratas. Revista Mexicana de Análisis de la Conducta, 31, 67-84, available via: http://dx.doi.org/10.5514/rmac.v31.i1.23241

Lotter, E. C., Woods, S. C., \& Vasseli, J. R. (1973). Schedule-induced polydipsia: An artifact. Journal of Comparative and Physiological Psychology, 83, 478-484, available via: http://dx.doi.org/10.1037/h0034670

Madden, G. J., Smethells, J. R., Ewan, E. E., \& Hursh, S. R. (2007). Tests of behavioral- 
economic assessments of relative reinforcer efficacy II: Economic complements. Journal of the Experimental Analysis of Behavior, 88, 355-367, available via: http://dx.doi.org/10.1901/jeab.2007.88-355

Pellón, R., \& Blackman, D. E. (1987). Punishment of Schedule-induced drinking in rats by signaled and unsignaled delays in food presentation. Journal of the Experimental Analysis of Behavior, 48, 417-434, available via: http://dx.doi. org/10.1901/jeab.1987.48-417

Reid, A. K., \& Staddon, J. E. R. (1982). Schedule-induced drinking: Elicitation, anticipation or behavioral interaction? Journal of the Experimental Analysis of Behavior, 38, 1-18, available via: http://dx.doi.org/10.1901/jeab.1982.38-1

Robinson, J. K., \& Flory, R. K. (1989). The control of schedule-induced polydipsia by meal size: Interaction with prior within-session eating and drinking. The Psychological Record, 39, 439-445.

Roca, A., \& Bruner, C. A. (2003). Efectos de la frecuencia de reforzamiento sobre el palanqueo en ratas privadas de comida. Revista Mexicana de Análisis de la Conducta, 29, 119-130.

Roca, A., \& Bruner, C. A. (2007). Intromisión de una sesión de beber inducido por el programa en un período de 24 horas. Revista Mexicana de Análisis de la Conducta, 33, 61-78, available via: http://dx.doi.org/10.5514/rmac.v33.i1.16267

Roper, T. J., \& Nieto, J. (1979). Schedule-induced drinking and other behavior in the rat, as a function of body weight deficit. Physiology and Behavior, 23, 673-678, available via: http://dx.doi.org/10.1016/0031-9384(79)90159-8

Rosenblith, J. Z. (1970). Polydipsia induced in the rat by a second-order schedule. Journal of the Experimental Analysis of Behavior, 14, 139-144, available via: http://dx.doi.org/10.1901/jeab.1970.14-139

Ruiz, J. A., \& Bruner, C. A. (2008). Demora de reforzamiento con agua en un procedimiento de beber inducido por el programa. Revista Mexicana de Análisis de la Conducta, 34, 97-109, available via: http://dx.doi.org/10.5514/rmac.v34.i1.16236

Segal, E. F., Oden, D. L., \& Deadwyler, S. A. (1965). Determinants of polydipsia: IV. Free reinforcement schedules. Psychonomic Science, 3, 11-12.

Staddon, J. E. R. (1977). Schedule-Induced Behavior. En W. K. Honig \& J. E. R. Staddon (Eds.), Handbook of Operant Behavior (pp. 125-152). Englewood Cliffs, NJ: Prentice-Hall.

Stein, L. (1964). Excessive drinking in the rat: Superstition or thirst? Journal of Comparative and Physiological Psychology, 58, 237-242.

Timberlake, W. (1997). An animal-centered, causal system approach to the understanding and control of behavior. Applied Animal Behaviour Science, 53, 107-129, available via: http://dx.doi.org/10.1016/S0168-1591(96)01154-9

Timberlake, W. (2000). Motivational modes in behavior systems. En R. R. Mowrer \& S. B. Klein (Eds.), Handbook of Contemporary Learning (pp. 155-209). Hillsdale, NJ: Erlbaum Associates.

Verplanck, W. S., \& Hayes, J. R. (1953). Eating and drinking as a function of main- 
tenance schedule. Journal of Comparative and Physiological Psychology, 47, 327- 333, available via: http://dx.doi.org/10.1037/h0055380

Wetherington, C. L. (1979). Schedule-induced drinking: Rate of food delivery and Herrnstein's equation. Journal of the Experimental Analysis of Behavior, 32, 323333, available via: http://dx.doi.org/10.1901/jeab.1979.32-323

Wetherington, C. L. (1982). Is adjunctive behavior a third class of behavior? Neuroscience \& Biobehavioral Reviews, 6, 329-350, available via: http://dx.doi. org/10.1016/0149-7634(82)90045-8 